\title{
Medición del potencial recurso eólico en los distritos de Huancavelica, Paucarbamba, Pampas, Acobamba y Lircay
}

\author{
Measurement of the potential wind resource in the districts of Huancavelica, Paucarbamba, \\ Pampas, Acobamba and Lircay \\ ${ }^{1, \mathrm{a}}$ Steve Brian Escobar Gaspar \\ ${ }^{2, \mathrm{~b}}$ Sayury Fanny Esplana Esteban \\ ${ }^{3, \mathrm{c}}$ Adiel Alvarez Ticllasuca \\ ${ }^{4, \mathrm{~d}}$ Fernando Martin Toribio Román
}

\begin{abstract}
ARTÍCULO ORIGINAL
${ }^{1}$ Universidad Nacional de Huancavelica. Huancavelica, Perú. E-mail: stevebrian92@gmail.com

${ }^{2}$ Universidad Nacional de Huancavelica. Huancavelica, Perú. E-mail: sayury.esplana.30@gmail.com

${ }^{3}$ Universidad Nacional Autónoma de Tayacaja "Daniel Hernández Morillo". Huancavelica, Perú. E-mail: adielalvarez@unat.edu.pe

${ }^{4}$ Universidad Nacional de Huancavelica. Huancavelica, Perú. E-mail: toribioroman@gmail.com
\end{abstract}

${ }^{\mathrm{a} O R C I D: 0000-0001-5297-5641}$

'ORCID: 0000-0002-2412-5591

'ORCID: 0000-0002-5410-0571

${ }^{\mathrm{d} O R C I D}$ 0000-0003-1139-9232

Palabras clave: Aerogeneradores, energías renovables, potencial eólico.

Keywords: Wind turbines, renewable energies, wind potential.

\section{Información adicional}

Presentado: 30/09/2020

Aprobado: 28/11/2020

\section{RESUMEN}

La energía eólica es una forma de energía limpia o renovable producida a partir del viento, es decir, una energía cinética producida por el efecto de las corrientes de aire. La investigación tuvo como objetivo analizar la cantidad de energía eléctrica que se genera a partir del potencial eólico en la región de Huancavelica. El instrumento que se utilizó fue la ficha de registro de datos. El análisis se realizó en cinco distritos de la región; para el análisis inferencial se usó la prueba de Wilcoxon con $\mathrm{p}<0.05$ que indica que la velocidad del viento es superior a $2 \mathrm{~m} / \mathrm{s}$. Los datos de velocidad del viento que fueron registrados por las estaciones meteorológicas en la región Huancavelica durante el año 2017 son adecuados para la implementación de aerogeneradores de baja potencia ( 70 $\mathrm{KWh} / \mathrm{d}$ ). De la misma forma, se obtuvo que el comportamiento de los indicadores (velocidad de viento y dirección de viento) tienden a ser homogéneos o cíclicos en el distrito de Huancavelica, Paucarbamba, Pampas, Acobamba y Lircay de la región de Huancavelica en el período 2017.

\section{ABSTRACT}

Wind energy is a clean or renewable energy which is produced from the wind, it is a kinetic energy produced by the effect of air currents. The aim of the research was to analyze the amount of electrical energy that is generated from the wind potential in the Huancavelica Region. The instrument used was the data record sheet, the sample was made up of 5 districts of this Region. The statistical method of Kolmogorov Smirnov was used for the inferential analysis. The wind speed data that were recorded by the meteorological stations in the Huancavelica Region during the year 2017, are suitable for the implementation of low power wind turbines $(70 \mathrm{KWh} / \mathrm{d})$. In the same way, it was obtained that the behavior of the indicators (wind speed and wind direction), tend to be homogeneous or cyclical in the district of Huancavelica, Paucarbamba, Pampas, Acobamba and Lircay of the Huancavelica Region in the period 2017. 


\section{INTRODUCCIÓN}

El área energía es uno de los más importantes responsables del cambio climático, gracias a la monumental proporción de gases de efecto invernadero que se crean por la quema de combustibles fósiles y las emisiones fugitivas (André et al., 2012). Gracias a los hechos expuestos anteriormente, la indagación científica y el desarrollo tecnológico han recibido un monumental fomento internacional, primordialmente luego del último informe del IPCC (Panel Intergubernamental para el Cambio Climático) publicado en febrero de 2007 (Pachauri \& Reisinger, 2007). Así, las energías renovables (solar, hidráulica, geotérmica, mareomotriz, biomasa, y eólica) surgen como alternativas a las fuentes convencionales de generación eléctrica (carbón, petróleo y gas natural), pues no emiten GEI durante su operación, mas sí durante su lapso de vida (Bojorquez, 2018).

Entre las fuentes de energía "limpia" - fuentes de energía que no provocan la emisión de gases de efecto invernadero (GEI) - se ha destacado la energía mecánica contenida en el viento, que demuestra el potencial de contribuir significativamente en el cumplimiento de los requisitos necesarios en cuanto a los costes de producción, seguridad del suministro y sostenibilidad medioambiental (Ulacia, 2007). Las energías renovables se encuentran en un nivel de desarrollo adecuado para su explotación comercial, una de ellas es la energía eólica que ha destacado por ser la más rentable (Marcos et al., 2015). La energía eólica ha crecido en los últimos años exponencialmente, este crecimiento demanda material, maquinaria, mano de obra y personal capacitado, al mismo ritmo del crecimiento, por lo que es necesario desarrollar en lo posible temas de energía eólica (Trujillo, 2014).

El desarrollo de la energía eólica en escala mundial es notable, representando una parte importante de la capacidad eléctrica global con fuentes renovables (Beltrán-Telles et al., 2017). La industria eólica mundial enfoca su atención hacia países en desarrollo con locaciones idóneas para la instalación de centrales eólicas (Juarez-Henandez \& León, 2014). La preocupación medioambiental y la necesidad de optimizar costes y aumentar la seguridad energética en muchos ámbitos de la actividad económica son responsables del creciente interés por conocer los aspectos técnicos y científicos asociados al uso de fuentes de energía renovables (Martins et al., 2008).

No obstante, para hacer uso del recurso eólico y maximizar la producción de energía eléctrica se deben tener en cuenta las condiciones cambiantes del viento, tanto la rapidez como la dirección, propiedades del viento (turbulencia) y densidad del aire; debido a que si no lo hacemos, todos los esfuerzos para capturar la más grande porción viable de viento podrían ser en vano (Trujillo, 2014). En el contexto actual de reconversión de la base energética, el viento es una buena fuente de energía que se viene aprovechando en muchos países del mundo. Es una fuente de energía renovable que se utiliza para la generación de electricidad, el bombeo de agua y muchas otras actividades (De Sales et al., 2019). La creciente demanda nacional y el desabastecimiento de energía eléctrica en algunas zonas del territorio peruano, especialmente en las zonas rurales, sumado al gran potencial eólico del Perú, en lugares como Ica, Cajamarca, Lambayeque, Piura, La Libertad, etc., representan un escenario ideal para atraer inversionistas nacionales y extranjeros que propicien el desarrollo del sector de la generación de energía eólica en el país (Calderón et al., 2017).

La presente investigación trata sobre la estimación del potencial eólico para la generación de energía eléctrica en los distritos de Huancavelica, Paucarbamba, Pampas, Acobamba y Lircay de la región de Huancavelica en el período 2017.

\section{MATERIAL Y MÉTODOS}

La recolección y procesamiento de datos se realizó durante los meses del año 2017, con la información de los distritos de Huancavelica, Paucarbamba, Pampas, Acobamba y Lircay de la región Huancavelica. La investigación fue de tipo aplicada, nivel descriptivo, se usó el método científico y el diseño no experimental 
(Morales, 2011). El instrumento utilizado fue la ficha de registro de datos (De Paz, 2008), teniendo como población la región Huancavelica, la muestra se conformó por 5 distritos de dicha región, el muestreo fue no probabilístico del tipo por conveniencia debido a la ubicación de las estaciones meteorológicas del Servicio Nacional de Meteorología e Hidrología (SENAMHI), para el análisis inferencial se usó la prueba de Wilcoxon con una significación del $5 \%$.

\section{Selección de estaciones meteorológicas del SENAMHI}

Para definir las estaciones meteorológicas del SENAMHI, se tuvieron en cuenta los siguientes aspectos: deben estar funcionando, deben ser estaciones convencionales porque se utilizaron promedios diarios, deben tener datos históricos de velocidad del viento, dirección del viento, temperatura y humedad relativa del año 2017, deben encontrarse a diferentes alturas sobre el nivel del mar (por lo menos $50 \mathrm{~m}$ de altura de diferencia entre estaciones del SENAMHI). El instrumento a utilizar fue la ficha de registros de datos. Definidas las estaciones meteorológicas del SENAMHI aptas para el estudio, se recolectaron los datos de velocidad y dirección del viento diario desde enero a diciembre del año 2017 que fueron proporcionadas de manera oficial por el SENAMHI.

\section{Técnicas de procesamiento y análisis de datos}

Para el procesamiento y análisis de datos de la potencia eólica disponible, los datos fueron tratados mediante estadística descriptiva. Previamente se realizó la prueba de normalidad Kolmogorov - Smirnov y por último la prueba de hipótesis con la prueba de Wilcoxon a una significancia de $5 \%$, para analizar si es adecuada la cantidad de energía eléctrica y establecer la velocidad promedio mensual, frecuencia y dirección predominante como parámetros más importantes para determinar con exactitud la orientación.

\section{Análisis mensual multianual de la velocidad del viento}

Se analizaron los valores promedios mensuales multianuales de velocidad del viento obtenidos a partir de las estaciones de monitoreo del viento del SENAMHI, durante el 2017. En base a la información promedio diaria de velocidad del viento, se elaboró un archivo en formato Excel donde se organizaron los valores promedio diarios de velocidad del viento para los períodos analizados entre los meses de enero a diciembre del 2017.

Para el cálculo de la velocidad del viento media, máxima, mínima, moda, mediana y desviación estándar del período analizado, se tomaron los datos diarios de cada mes analizado y se hallaron los valores mensuales para el período analizado.

\section{Análisis mensual multianual de la temperatura, humedad relativa y presión atmosférica}

Se analizaron los datos promedios mensuales multianuales de temperatura, humedad relativa de las estaciones seleccionadas con el fin de establecer una relación de dicha variable con los datos de velocidad el viento. Tomando las alturas sobre el nivel del mar, por medio del cálculo de presión atmosférica para cada una de las estaciones seleccionadas (Murcia \& Lesmes, 2010).

Presión atmosférica

Donde:

$$
P=P_{0} \times\left(e^{\alpha * h}\right)
$$

$\mathrm{P}=$ Presión atmosférica $(\mathrm{Pa})$.

$\mathrm{P}_{\mathrm{o}}=$ Presión atmosférica a nivel del mar $(101325 \mathrm{~Pa})$.

$\mathrm{h}=$ Altura sobre el nivel del mar (m s. n. m.).

$\alpha=$ Constante atmosférica $\left(1.18 * 10^{\wedge}(-4) \mathrm{m}^{\wedge}(-1)\right)$ 


\section{Estimación de la densidad del aire}

Para el desarrollo de proyectos de instalación de aerogeneradores es necesario conocer la velocidad del viento a la altura del eje del rotor, habitualmente ubicado en la torre elevada, por lo que es necesario inferir la ecuación de la velocidad del viento a diferentes alturas mediante el siguiente método (Carta et al., 2009).

Densidad del aire

$$
\rho=\frac{0.348444 \times \mathrm{P}-\mathrm{h}_{\mathrm{r}}(0.00252 \mathrm{~T}-0.020582)}{273.15+\mathrm{T}}
$$

Donde:

$\rho=$ Densidad del aire $\left((\mathrm{Kg}) /\left(\mathrm{m}^{3}\right)\right)^{\circ}$

$\mathrm{P}=$ Presión atmosférica $(\mathrm{hPa})$

$\mathrm{T}=$ Temperatura termodinámica del aire $\left({ }^{\circ} \mathrm{C}\right)$

$\mathrm{h}_{\mathrm{r}}=$ Humedad relativa $(\%)$.

\section{Densidad de potencia en función de la densidad del aire}

La estimación de la densidad de potencia eólica específica se realizó de acuerdo a la ecuación de densidad de potencia eólica (García, 2016).

Densidad de potencia eólica

$$
\frac{P_{\text {inst }}}{A}=\frac{1}{2} \rho\left(v^{3}\right)_{\text {inst }}
$$

Donde:

$\frac{P_{\text {inst }}}{\mathrm{A}}=$ Densidad de potencia eólica $\left(\right.$ watt $\left./ \mathrm{m}^{2}\right)$

$\rho=$ Densidad del aire $\left(\mathrm{Kg} / \mathrm{m}^{3}\right)$

$\mathrm{v}=$ velocidad del viento $(\mathrm{m} / \mathrm{s})$

Para realizar el ajuste de los datos se organizaron las velocidades del viento registradas en intervalos constantes de $1 \mathrm{~m} / \mathrm{s}$, para posteriormente calcular la probabilidad de Weibull de cada una de las velocidades.

Se realizó el cálculo de la energía eléctrica $(\mathrm{kWh})$ que podría obtenerse potencialmente con el equipo seleccionado (Yglesias, 2015) para un aerogenerador de baja potencia (100kw) en base a los datos de curva potencia y la probabilidad de Weibull.

\section{RESULTADOS}

La velocidad máxima del viento fue $10 \mathrm{~m} / \mathrm{s}$, registrado en el mes de agosto; y el valor mínimo de velocidad fue $3 \mathrm{~m} / \mathrm{s}$ en los meses de marzo, abril, mayo, setiembre, octubre, noviembre y diciembre (Figura 1). La velocidad promedio del viento se ubica en el rango de 5.2 a $7.29 \mathrm{~m} / \mathrm{s}$. 


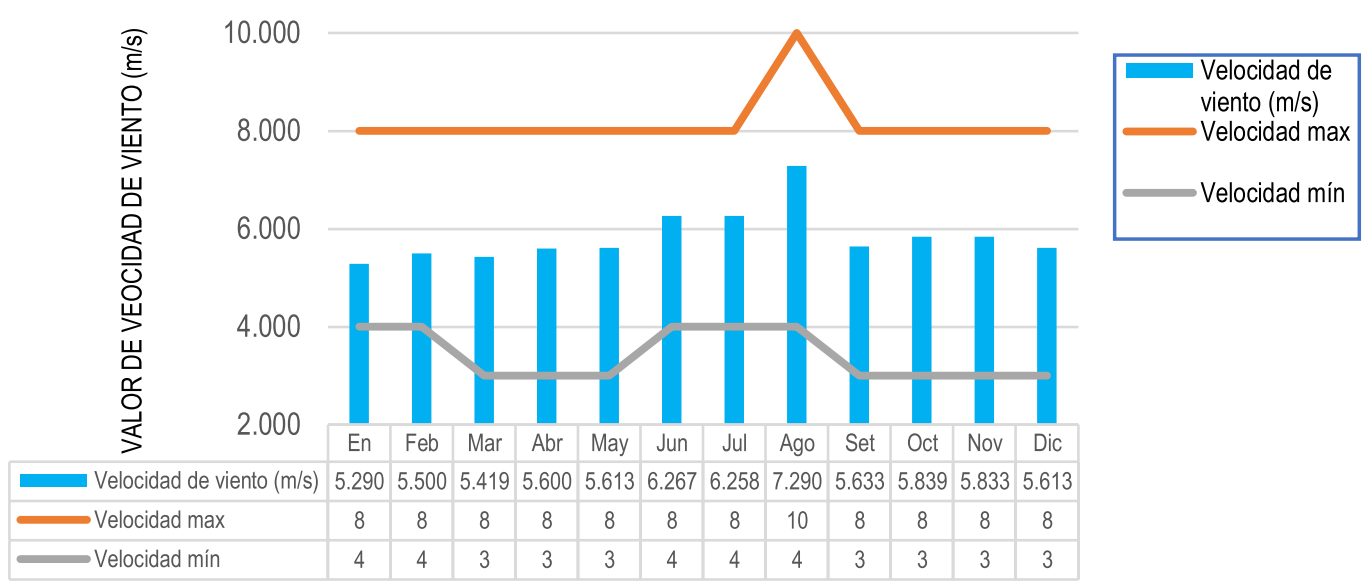

Figura 1. Velocidad del viento para el año 2017- Estación meteorológica de Huancavelica

La mayor potencia generada fue $1602.60 \mathrm{KW}$, registrada en el mes de agosto; y el menor valor de potencial generado fue $650.70 \mathrm{KW}$ en el mes de enero (Figura 2). Las viviendas en la ciudad de Huancavelica son consideradas de tipo socioeconómico "C", las cuales requieren $277.87 \mathrm{KW} / \mathrm{mes} /$ vivienda de energía eléctrica (según INEI). Entonces, la mayor potencia generada (1602.60 KW) abastecería a un promedio de cinco (05) viviendas, mientras que el menor valor de potencia generada $(650.70 \mathrm{KW})$ abastecería a un promedio de dos (02) viviendas. Por ello, si se desea abastecer a toda la población de Huancavelica se debe incrementar el número de aerogeneradores en proporción al número de viviendas.

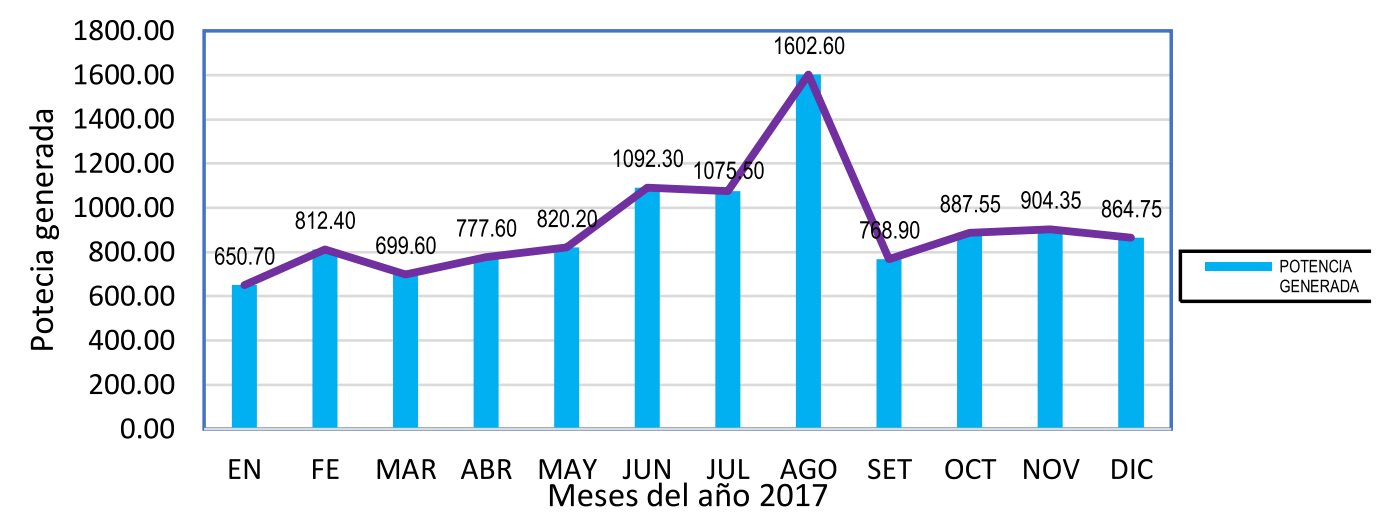

Figura 2. Potencia generada en la ciudad de Huancavelica - año 2017

La velocidad máxima fue $10 \mathrm{~m} / \mathrm{s}$ registrado en los meses de enero, febrero, mayo, junio, julio, agosto, setiembre, octubre y noviembre; mientras que el valor mínimo fue $3 \mathrm{~m} / \mathrm{s}$ en los meses de enero, marzo, abril, junio, setiembre, octubre, noviembre y diciembre. Además, la velocidad promedio del viento se ubica en el rango de 4.7 a $8 \mathrm{~m} / \mathrm{s}$ (Figura 3 ). 


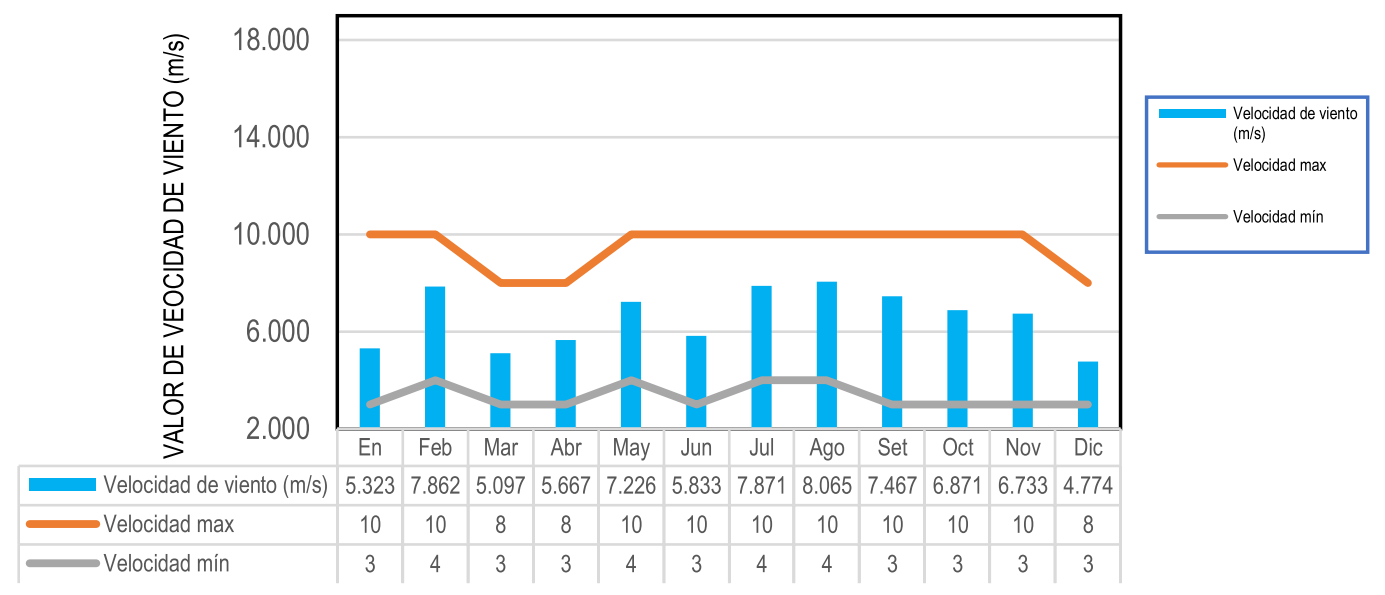

Figura 3. Velocidad del viento para el año 2017-Estación meteorológica de Paucarbamba.

La mayor potencia generada fue $2127.75 \mathrm{KW}$, registrada en el mes de agosto; y el menor valor de potencial generado fue $717.75 \mathrm{KW}$ en el mes de marzo (Figura 4). Las viviendas en la ciudad de Paucarbamba son consideradas de tipo socioeconómico "E", las cuales requieren $52.95 \mathrm{KW} / \mathrm{mes} /$ vivienda de energía eléctrica (según INEI). Entonces, la mayor potencia generada $(2127.75 \mathrm{KW}$ ) abastece a un promedio de cuarenta (40) viviendas, mientras que el menor valor de potencia generada $(717.75 \mathrm{KW})$ abastece a un promedio de trece (13) viviendas. Por lo que, si se desea abastecer a toda la población de Paucarbamba, se debe incrementar el número de aerogeneradores en proporción al número de viviendas.

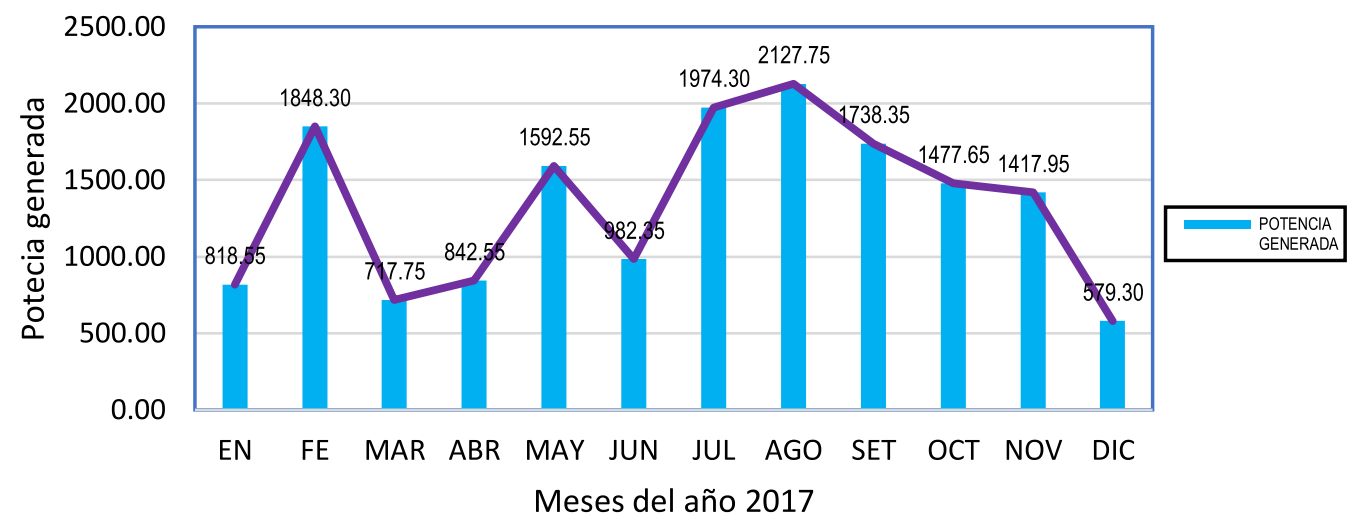

Figura 4. Potencia generada en la ciudad de Paucarbamba - año 2017

La velocidad máxima viene a ser igual a $10 \mathrm{~m} / \mathrm{s}$, registrado en los meses de enero, febrero, mayo, agosto, setiembre y diciembre. Por otra parte, el valor mínimo de velocidad fue $3 \mathrm{~m} / \mathrm{s}$ en los meses de abril, mayo, junio, setiembre y octubre. La velocidad promedio del viento se ubica en el rango de 6.2 a $7.5 \mathrm{~m} / \mathrm{s}$ (Figura 5). 


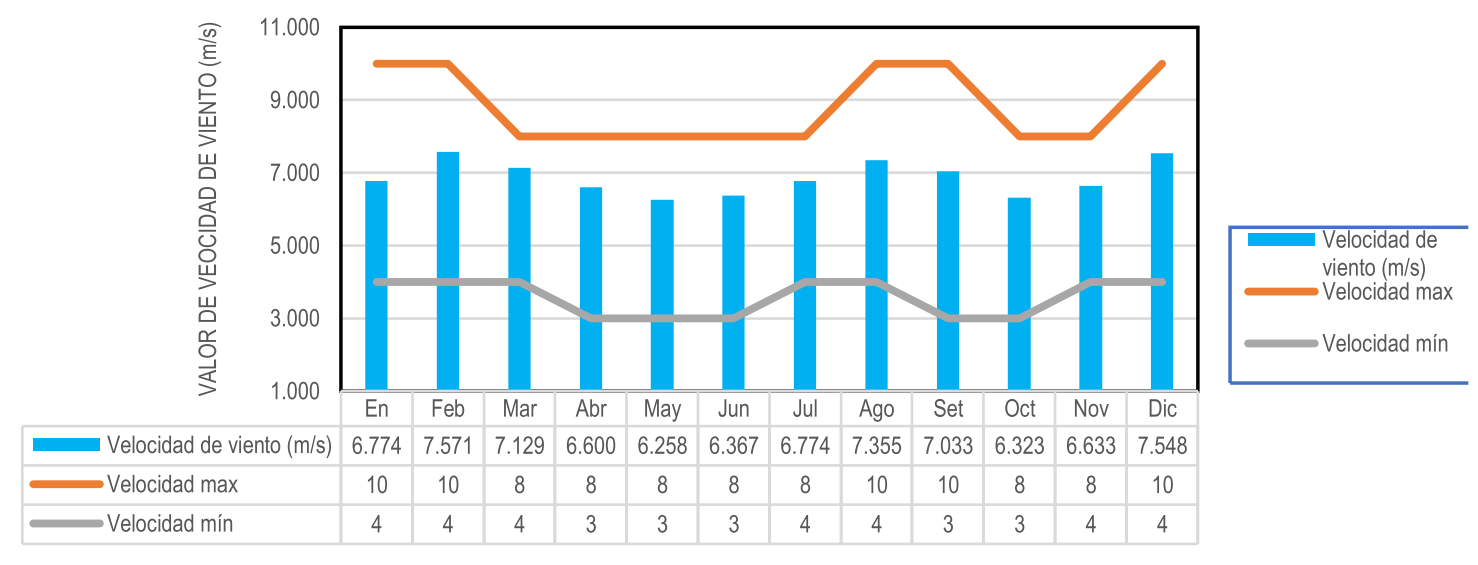

Figura 5. Velocidad del viento para el año 2017-Estación meteorológica de Pampas.

La mayor potencia generada fue $1724.85 \mathrm{KW}$, registrada en el mes de diciembre; y el menor valor de potencial generado fue 1095.90 KW en el mes de octubre (Figura 6). La ciudad de Pampas es considerada de tipo socioeconómico “D”, las cuales requieren $129.55 \mathrm{KW} / \mathrm{mes} /$ vivienda de energía eléctrica (según INEI). Entonces, la mayor potencia generada $(1724.85 \mathrm{KW})$ abastecería a un promedio de trece (13) viviendas, mientras que el menor valor de potencia generada $(1095.90 \mathrm{KW})$ abastecería a un promedio de ocho (08) viviendas. Por lo que, si se desea abastecer a toda la población de Pampas, se debe incrementar el número de aerogeneradores en proporción al número de viviendas.

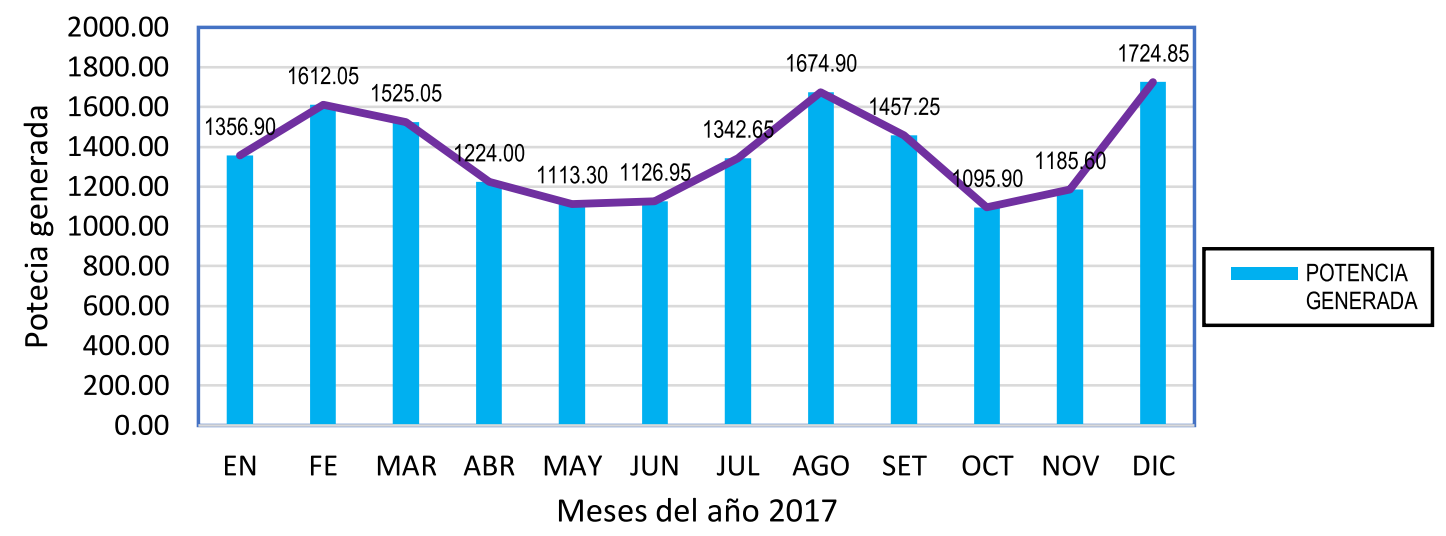

Figura 6. Potencia generada en la ciudad de Pampas - año 2017

La velocidad máxima fue $10 \mathrm{~m} / \mathrm{s}$, registrada en los meses de octubre y noviembre; y el valor mínimo de velocidad fue $3 \mathrm{~m} / \mathrm{s}$ registrado en los doce meses del año 2017. La velocidad promedio del viento se ubica en el rango de 3.6 a $4.7 \mathrm{~m} / \mathrm{s}$ (Figura 7 ). 


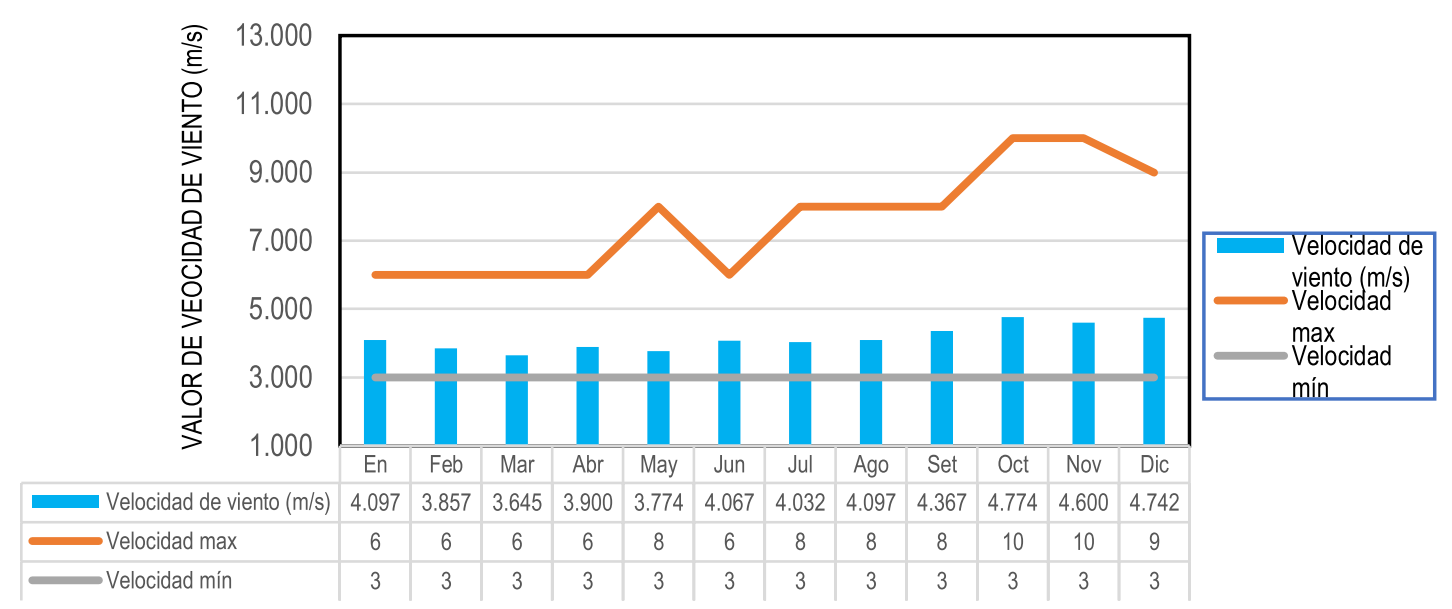

Figura 7. Velocidad del viento para el año 2017-Estación meteorológica de Acostambo

La mayor potencia generada fue $579.90 \mathrm{KW}$, registrada en el mes de diciembre; y el menor valor de potencial generado fue $192.60 \mathrm{KW}$ en el mes de marzo (Figura 8). Las viviendas en la ciudad de Acostambo son consideradas de tipo socioeconómico "E", las cuales requieren $52.95 \mathrm{KW} / \mathrm{mes}$ de energía eléctrica (según INEI). Entonces, la mayor potencia generada (579.90 KW) abastecería a un promedio de diez (10) viviendas, mientras que el menor valor de potencia generada $(192.60 \mathrm{KW})$ abastecería a un promedio de tres (03) viviendas. Por lo cual, si se desea abastecer a toda la población de Acostambo se debe incrementar el número de aerogeneradores en proporción al número de viviendas.

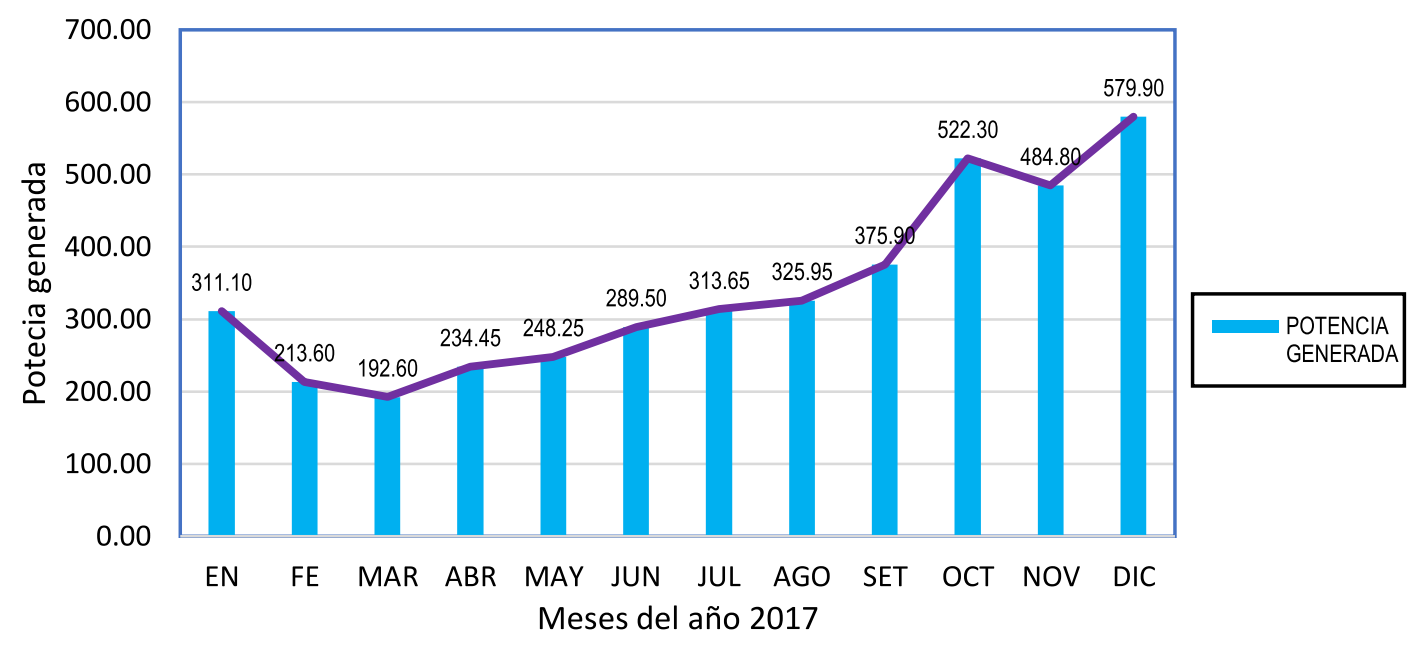

Figura 8. Potencia generada en la ciudad de Acostambo - año 2017

La velocidad máxima fue $6 \mathrm{~m} / \mathrm{s}$, registrada en los meses de agosto y noviembre; y el valor mínimo de velocidad fue $3 \mathrm{~m} / \mathrm{s}$ registrado en los doce meses del año 2017. La velocidad promedio del viento se ubica en el rango de 3 a $4 \mathrm{~m} / \mathrm{s}$ (Figura 8). 


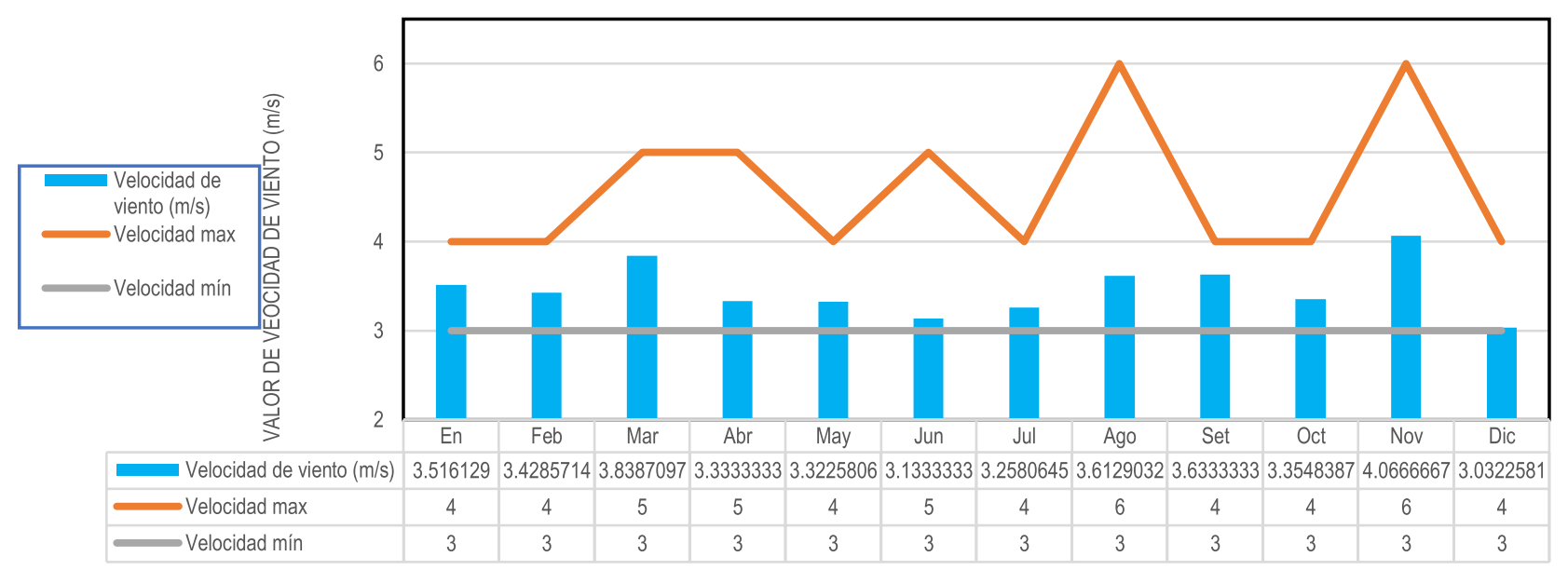

Figura 9. Velocidad del viento para el año 2017-Estación meteorológica de Lircay

La mayor potencia generada fue $240.90 \mathrm{KW}$, registrada en el mes de noviembre; y el menor valor de potencial generada fue de $60.60 \mathrm{KW}$ en el mes de junio (Figura 10). Las viviendas en la ciudad de Lircay son consideradas de tipo socioeconómico "E", las cuales requieren $52.95 \mathrm{KW} / \mathrm{mes}$ de energía eléctrica (según INEI). Entonces, la mayor potencia generada $(240.90 \mathrm{KW})$ abastecería a un promedio de cuatro (04) viviendas, mientras que el menor valor de potencia generada $(60.60 \mathrm{KW})$ abastecería a un promedio de una (01) vivienda. Por lo cual, si se desea abastecer a toda la población de Lircay se deben de incrementar el número de aerogeneradores en proporción al número de viviendas.

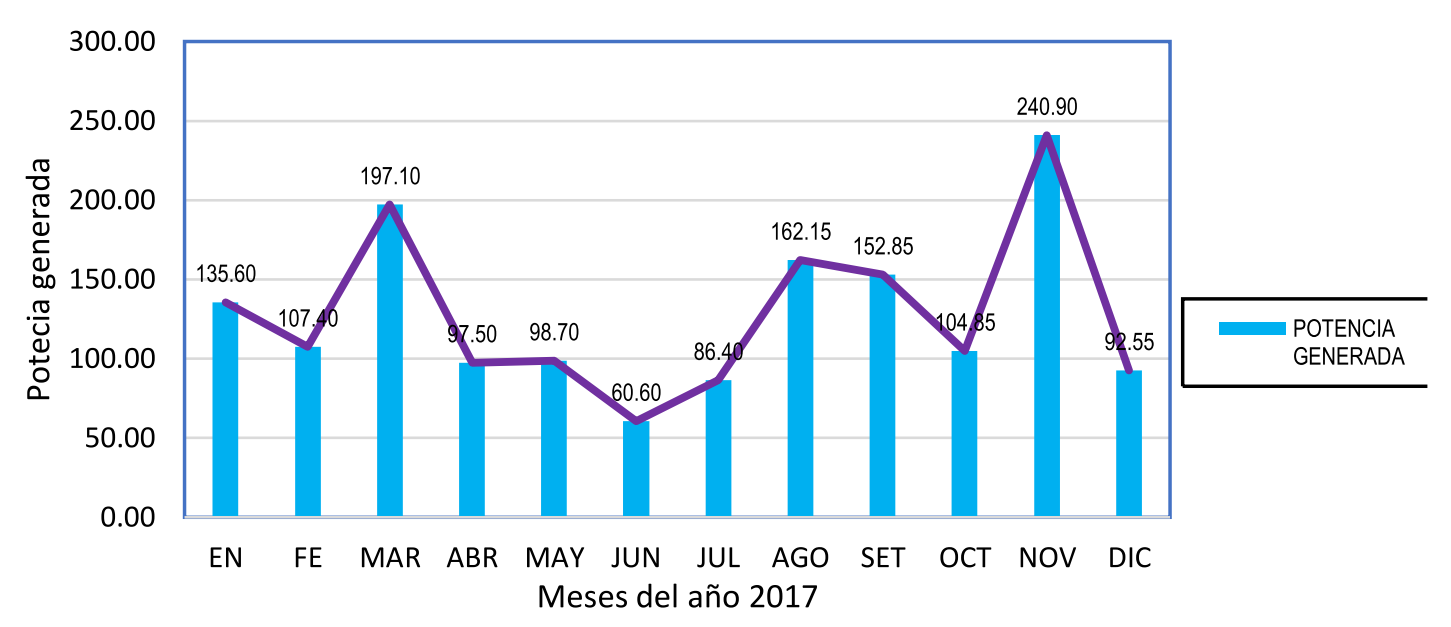

Figura 10. Potencia generada en la ciudad de Lircay - año 2017 


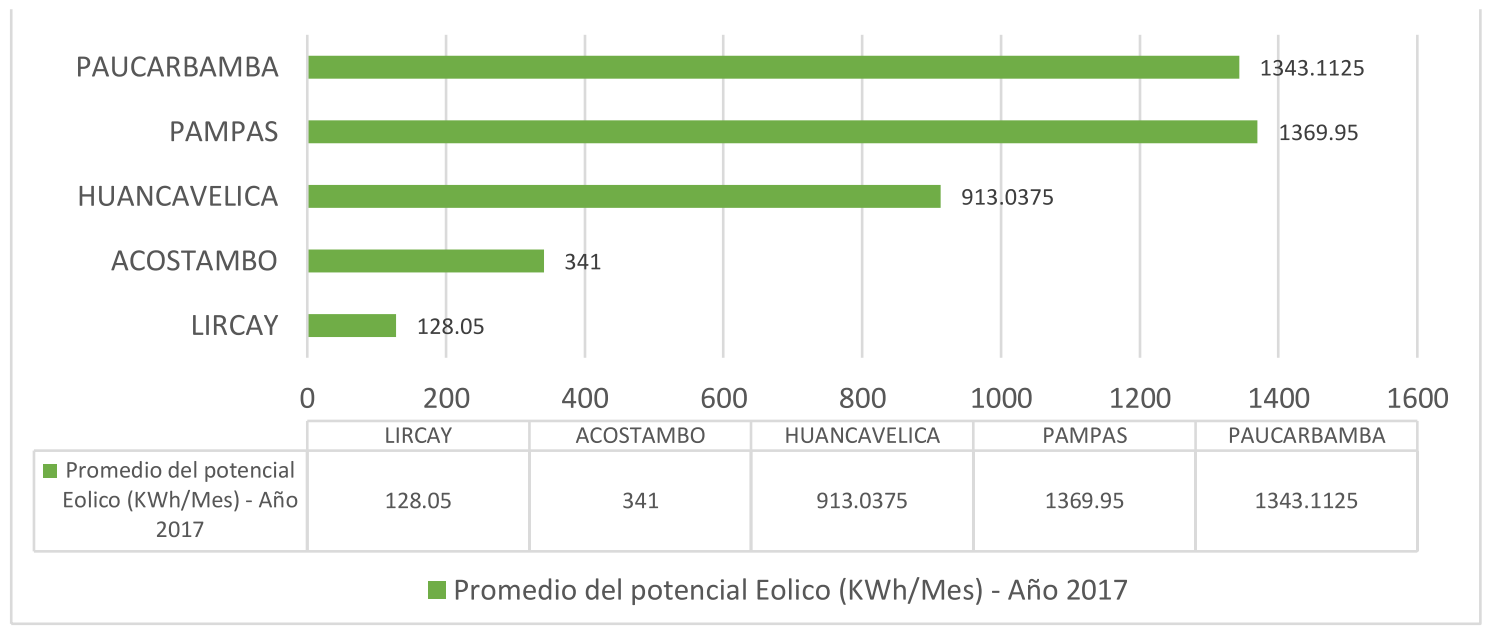

Figura 11. Promedio del potencial Eólico (KWh/Mes) de los diferentes distritos, en el 2017

El distrito con mayor potencia eólica fue Pampas, llegando a los $1369.95 \mathrm{KWh} / \mathrm{mes}$; y el distrito con menor potencia fue Lircay, mostrando un valor de $127.22 \mathrm{KWh} / \mathrm{mes}$ (Figura 11).

\section{DISCUSIÓN}

La investigación demostró que es adecuada la cantidad de potencial eólico para la generación de energía eléctrica en la región de Huancavelica en el período estudiado. Los resultados muestran velocidades de viento promedio, que oscilan entre 3 y $10 \mathrm{~m} / \mathrm{s}$. Debido a la geografía y la altitud de la región, los datos recolectados por cada estación, fueron suficientemente adecuados para definir una respuesta positiva a la implementación y uso del mencionado recurso. De la misma forma, se tiene que Tafur \& Pizza (2014) en su investigación obtuvo que las velocidades de viento adecuadas estuvieron entre 3 y $25 \mathrm{~m} / \mathrm{s}$. Otros estudios como el de Avellaneda (2012) y el de Arnes (2014) indican velocidades promedio de $5 \mathrm{~m} / \mathrm{s}$, adecuadas para producir energía eólica.

Hernández-Escobedo et al. (2012) en su estudio obtuvo velocidades de viento que oscilan entre 5.5 y 6.2 $\mathrm{m} / \mathrm{s}$, registradas en diversas estaciones meteorológicas; así como Buñuelos (2012), en el trabajo de investigación que desarrolló, recolectó datos de velocidad de viento a partir de los $4 \mathrm{~m} / \mathrm{s}$ hasta $20 \mathrm{~m} / \mathrm{s}$ de una estación meteorología propia. Como se puede deducir, una velocidad a partir de $10 \mathrm{~m} 4 \mathrm{~m} / \mathrm{s}$ es adecuada para la generación de energía eólica suficiente para hacer funcionar un aerogenerador de baja potencia y proveer de energía eléctrica a zonas andinas que no cuentan con la red de energía eléctrica convencional como el caso de las zonas rurales de Huancavelica.

Hernández (2012) en su trabajo de investigación utilizó un aerogenerador modelo AW 70/1500 Class I, el cual registró datos de velocidad de viento de 5.1 a $13.6 \mathrm{~m} / \mathrm{s}$. Por lo que se mantiene la teoría, entre los trabajos de investigación ya señalados, que muestran rangos de acción adecuados para producir un potencial eólico óptimo y así generar energía eléctrica.

En las zonas estudiadas las velocidades de viento superan $\operatorname{los} 2 \mathrm{~m} / \mathrm{s}$, que refieren las investigaciones revisadas como las que ofrecen un potencial para usar la energía eólica, información que se respalda con lo mencionado en la ficha técnica E70PRO, donde se indican las características técnicas, eléctricas y de funcionamiento del aerogenerador de capacidad de $70 \mathrm{KWh} /$ día, dando hincapié a que el arranque de dicho aerogenerador se da con velocidades de viento igual a $2 \mathrm{~m} / \mathrm{s}$ y dicho aerogenerador es eficiente cuando hay presencia de viento en un rango de 2 a $60 \mathrm{~m} / \mathrm{s}$. 


\section{CONCLUSIONES}

Las velocidades de viento registradas en las estaciones oscilan entre 3 y $10 \mathrm{~m} / \mathrm{s}$, por lo que la cantidad de energía eléctrica generada a partir del potencial eólico es adecuada para un aerogenerador de baja potencia de capacidad de $70 \mathrm{kwh} /$ día.

De acuerdo con las evidencias e información presentadas, el que presenta mayor potencial eólico es la ciudad de Pampas con $1369.95 \mathrm{KWh} / \mathrm{mes}$, seguida por las ciudades de Paucarbamba, Huancavelica, Acostambo y Lircay con $1343.1125 \mathrm{KWh} / \mathrm{mes}, 913.0375 \mathrm{KWh} / \mathrm{mes}$ y $341 \mathrm{KWh} / \mathrm{mes}, 128.05 \mathrm{KWh} / \mathrm{mes}$ respectivamente. Como consecuencia de la escasa infraestructura actualmente disponible, resulta necesario mejorar diferentes aspectos en la generación de energía renovable. Es necesario facilitar el diseño, construcción y operación de las formas de transmisión y distribución de energía provenientes de fuentes renovables como la energía eólica.

\section{REFERENCIAS}

André, F., Castro, L., \& Cerdá, E. (2012). Las energías renovables en el ámbito internacional. Cuadernos Económicos de ICE, (83), 11-36.

Ulacia, G. (2007). El efecto de la globalización en el sector eólico de las energías renovables. Una visión desde Gamesa. Boletín de Estudios Económicos, 62(192), 493-509.

Arnés, C. (2014). Estudio del potencial eólico para generación eléctrica en el itsmo de Tehuantepec, Oaxaca, México. Escuela Técnica de Ingenieros Industriales y Telecomunicación.

Avellaneda, A. (2012). Estudio del potencial de generación de energía eólica en la zona del páramo de chontales, municipios de Paipa y Sotaquirá. Departamento de Boyacá (tesis de posgrado). Universidad Libre.

Beltrán-Telles, A., Morera-Hernández, M., López-Monteagudo, F., \& Villela-Varela, R. (2017). Prospectiva de las energías eólica y solar fotovoltaica en la producción de energía eléctrica. CienciaUAT, 11(2), 105. https://doi.org/10.29059/cienciauat.v11i2.742

Bojorquez, M. (2018). Planeamiento de un parque eólico marino en la costa peruana: regiones de Ica, Piura y La Libertad (tesis de pregrado). Pontificia Universidad Católica del Perú.

Buñuelos, F. (2011). Impacto de la generación eléctrica usando fuentes de energía eólica en la red eléctrica nacional. Instituto de Ingeniería. Universidad Autónoma de México.

Calderón, R., Leyva, V., Miranda, M., \& Pais, C. (2017). Planeamiento Estratégico para el Sector de Energía Eólica del Perú (tesis de pregrado). Pontificia Universidad Católica del Perú.

Carta, J., Ramirez, P., \& Velázquez, S. (2009). Una revisión de las distribuciones de probabilidad de la velocidad del viento utilizadas en el análisis de la energía eólica: estudios de caso en las Islas Canarias. Revisiones de energías renovables y sostenibles, 13 (5), 933-955.

De Sales, M., De Sales, M., \& Tavares, M. (2019). Impactos Socioeconômicos, Ambientais e Tecnológicos Causados pela Instalação dos Parques Eólicos no Ceará. Revista Brasileira de Meteorologia, 34(3), 399-411. https://doi.org/10.1590/0102-7786343049

De Paz, D. (2008). Conceptos y técnicas de recolección de datos en la investigación jurídico social.

García, Á. (2016). La Enseñanza de las Energías Renovables. Revista Española de Física, 30(2), 33-36.

Hernández-Escobedo, Q., Espinosa-Arenal, F., Saldaña-Flores, R., \& Rivera-Blanco, C. (2012). Evaluación del potencial eólico para la generación de energía eléctrica en el estado de Veracruz, México. Dyna, 79(171).

Juárez-Hernández, S., \& León, G. (2014). Energía eólica en el istmo de Tehuantepec: desarrollo, actores y oposición social. Problemas del desarrollo, 45(178), 139-162.

Marcos, A., Martínez de Salazar, E., Fernández, J., Gañan, J., Turegano, J., \& Piñero, J. (2015). Consideraciones al proyecto de energía eólica. Parámetros que influyen en su rentabilidad. 
Martins, F., Guarnieri, R., \& Pereira, E. (2008). O aproveitamento da energia eólica. Revista Brasileira de Ensino de Física, 30 (1), 1304.1-1304.13

Morales, J. (2011). Análisis de los factores clave para la inversión en proyectos de energías renovables: el caso termosolar: la transición de un modelo económico" energívoro" a un modelo económico sostenible. Universidad Complutense de Madrid, Servicio de Publicaciones.

Murcia, J., \& Lesmes, H. J. Z. (2010). Algoritmo usado para estimar energía eólica en Colombia. Comisión Colombiana del Espacio-CCE Grupo de Observación de la Tierra.

Pachauri, R., \& Reisinger, A. (2007). Cambio climático 2007: Informe de síntesis. Contribución de los Grupos de trabajo I, II y III al Cuarto Informe de evaluación del Grupo Intergubernamental de Expertos sobre el Cambio Climático (IPCC).

Tafur, A., \& Pizza, A. (2014). Modelación y evaluación del potencial eólico como una fuente de generación de energía eléctrica en la zona costera de Cartagena (Doctoral dissertation). Universidad de Cartagena.

Tradavich, J. (2012). Curva de potencia de un aerogenerador. Vázquez, M. (2017). Potencia, 1, 6.

Trujillo, A. (2014). Evaluación del recurso eólico en la universidad tecnológica de ciudad Juárez (Doctoral dissertation). Centro de Investigación en Materiales Avanzados.

Yglesias, Y. (2015). Estudio técnico y económico de la instalación de aerogeneradores para suministrar energía eléctrica a las viviendas del centro poblado Playa Blanca-Piura. 